\title{
PLANTIO DE ESPÉCIES NATIVAS PARA RESTAURAÇÃO DE ÁREAS EM UNIDADES DE CONSERVAÇÃO: UM ESTUDO DE CASO NO SUL DO BRASIL
}

\author{
Suzane Bevilacqua Marcuzzo ${ }^{1}$, Maristela Machado Araújo ${ }^{2}$, Ezequiel Gasparin ${ }^{3}$ \\ ${ }^{1}$ Universidade Federal de Santa Maria, Departamento Multidisciplinar, Silveira Martins, Rio Grande do Sul, Brasil - \\ smarcuzzo@gmail.com \\ ${ }^{2}$ Universidade Federal de Santa Maria, Departamento de Ciências Florestais, Santa Maria, Rio Grande do Sul, Brasil - \\ araujo.maristela@gmail.com \\ ${ }^{3}$ Universidade Federal de Lavras, Programa de Pós-Graduação em Engenharia Florestal, Lavras, Minas Gerais, Brasil - \\ egasparin@gmail.com
}

Recebido para publicação: 17/07/2013 - Aceito para publicação: 13/08/2014

\begin{abstract}
Resumo
Este estudo buscou identificar espécies nativas potenciais para restauração de áreas degradadas em diferentes métodos de preparo de solo. O experimento foi implantado no Parque Estadual Quarta Colônia, RS, em blocos casualizados. No plantio, foi utilizado o modelo que considera espécies de preenchimento, juntamente com espécies de diversidade. Tratamentos: T1 - cova + plantio de espécies arbóreas (grupo de preenchimento - GP e de diversidade - GD); T2 - cova + plantio de espécies arbóreas + leguminosa (Vicia sativa); T3 - subsolagem + plantio de espécies arbóreas; e T4 - subsolagem + plantio de espécies arbóreas + leguminosa. As variáveis observadas, diâmetro do coleto, altura e mortalidade, foram submetidas a análise de variância. Para as 13 espécies arbóreas, as mesmas variáveis, além do diâmetro de copa, foram submetidas ao teste de Kruskal-Wallis. Constatou-se que o uso de subsolagem foi o melhor método, indiferentemente ao uso de leguminosa. Enterolobium contortisiliquum, Schinus terebinthifolius e Inga vera são indicadas para compor o grupo de preenchimento, enquanto que as espécies utilizadas para formar o grupo de diversidade devem ser introduzidas após a cobertura parcial do dossel, considerando necessitarem de ambiente mais favorável. Palavras-chave: Áreas protegidas; espécies arbóreas; grupos sucessionais; preparo de solo.
\end{abstract}

\begin{abstract}
Planting species to restore protected areas: a case study in Southern Brazil. This research aims to identify potential native species for restoration of degraded areas regarding different soil preparation techniques. The experiment was developed in the Quarta Colonia State Park, RS, in randomized blocks. In planting, it was applied the model that considers fast growth and diversity species groups. The treatments: T1 - hole + planting of tree species (fill group - GP and of diversity group - GD); T2 hole + tree species planting + legume (Vicia sativa); T3- subsoiling + tree species planting; and T4 subsoiling + tree species planting + legume. Observed variables, which include collect diameter, height and mortality, were submitted to analysis of variance. In relation to the 13 tree species, we submitted the same variables, plus crown diameter to the Kruskal -Wallis test. As result, subsoiling was the best method, indifferent to the use of legume. Enterolobium contortisiliquum, Schinus terebinthifolius and Inga vera are indicated to compose the fill group, while the species of the diversity group should be introduced after partial canopy coverage, considering they need more propitious environmental conditions.

Keywords: Protected area; successional groups; soil preparation.
\end{abstract}

\section{INTRODUÇÃO}

As Florestas Estacionais representam $42 \%$ das florestas no planeta e variam em distribuição e cobertura ao redor do mundo, devido à influência da sazonalidade climática (MURPHY; LUGO, 1986). Na região Sul do Brasil, essa fisionomia está associada ao clima de duas variações térmicas acentuadas, uma de cinco meses, com média de $20^{\circ} \mathrm{C}$, e outra de até dois meses, abaixo de $15^{\circ} \mathrm{C}$ (LEITE, 2002). 
Estudos em nível global (MILES et al, 2006), indicaram que as Florestas Estacionais são a fitofisionomia mais ameaçada do mundo, encontrando-se totalmente fragmentadas. Esse fato se comprova mediante a situação dos remanescentes florestais encontrados sobre as encostas do Planalto Meridional, na região central do Rio Grande do Sul, reduzidos e fragmentados, em virtude do desenvolvimento rural e urbano (KILCA; LONGHI, 2011).

Nesse sentido, as unidades de conservação são estratégias utilizadas para a manutenção da biodiversidade em áreas naturais remanescentes. Entretanto, o estabelecimento dessas áreas pode não ser suficiente para assegurar a manutenção de comunidades de fauna e flora ao longo do tempo, principalmente se tal prática estiver dissociada de uma abordagem que assegure a conservação de extensões mais abrangentes da paisagem (BRITEZ et al., 2003).

A restauração de ecossistemas florestais pode ser acelerada pelo método de plantio de espécies facilitadoras da sucessão natural, em locais onde uma série de barreiras, como geadas e compactação do solo, impedem o desenvolvimento desse processo. Contudo, é necessário levar em consideração na aplicação de modelos de restauração as características da fisionomia regional da Floresta Estacional, que determinam padrões distintos de resiliência, condições menos favoráveis ao crescimento vegetal, devido ao clima e importância relativa de grupos de dispersores, parâmetros que emergem como fatores controladores da velocidade sucessional da floresta (TABARELLI; MANTOVANI, 1999).

As espécies desejáveis para os plantios de restauração devem apresentar características como capacidade de estabelecimento em condições limitantes, atração da fauna, rápido crescimento e grande deposição de serapilheira (BARBOSA, 2000). De acordo com Rodrigues et al. (2010), a restauração de um ecossistema pode ser realizada por meio de grupos funcionais, de acordo com as fases do processo sucessional, a fim de se obter inicialmente uma rápida e boa cobertura de solo, eliminando a competição com herbáceas e gramíneas agressivas, além de criar um ambiente favorável para os indivíduos do grupo de diversidade. Tal grupo é constituído por muitas espécies com poucos indivíduos de cada espécie, havendo principalmente a presença daquelas comuns ao final do processo de sucessão vegetal.

Tendo em vista essas premissas, e visando assegurar o desempenho de um plantio que trate dessas peculiaridades, é necessário considerar que o crescimento das mudas é influenciado pelas condições de aeração, infiltração, umidade e nutrientes que o solo oferece ao sistema radicular. Nesse sentido, Gonçalves (2002) constatou que o preparo do solo interfere na taxa de sobrevivência e no posterior crescimento das plantas, por atuar diretamente sobre os fatores físicos, químicos e biológicos do solo, alterando a disponibilidade de recursos hídricos e nutricionais às plantas. A utilização de vegetação de cobertura pode melhorar expressivamente a qualidade do solo, tais como algumas espécies de leguminosas, por exemplo Vicia sativa L. (ervilhaca), que são forrageiras de inverno, com alta capacidade de fixação de nitrogênio atmosférico (GIACOMINI et al., 2003), nutriente essencial para o crescimento das mudas.

Assim, este trabalho teve por objetivo avaliar: a) se a subsolagem e o uso de leguminosa podem influenciar positivamente no desenvolvimento do modelo testado; e b) quais seriam as espécies do grupo de preenchimento e de diversidade com potencial de uso para restauração de áreas degradadas.

\title{
MATERIAL E MÉTODOS
}

\begin{abstract}
Área de estudo
O presente estudo foi realizado no Parque Estadual Quarta Colônia (P.E.Q.C.), uma unidade de conservação de proteção integral com área de 1.847,9 ha, cujas coordenadas geográficas da sede administrativa são $29^{\circ} 37^{\prime} 40.80^{\prime \prime} \mathrm{S}$ e $53^{\circ} 22^{\prime} 0.38^{\prime \prime} \mathrm{O}$.

O parque está localizado nos municípios de Agudo e Ibarama, Rio Grande do Sul, na bacia hidrográfica do rio Jacuí, caracterizando-se por apresentar encostas florestadas e áreas em processo de restauração, decorrente da obra da Usina Hidrelétrica (UHE) Dona Francisca.

O solo da região é classificado como Cambissolo Háplico Eutrófico típico (EMBRAPA, 2006). Segundo a classificação de Köppen, o clima da região é "Cfa", por apresentar temperatura média do mês mais frio entre -3 e $18{ }^{\circ} \mathrm{C}$ (junho a agosto), e do mês mais quente superior a $22{ }^{\circ} \mathrm{C}$, com chuvas bem distribuídas ao longo do ano (NIMER, 1990). A vegetação é integrante do bioma Mata Attântica, mais especificamente da região fitogeográfica de Floresta Estacional Decidual do Sul do Brasil (IBGE, 2012), também denominada de Floresta Estacional Subtropical (SCHUMACHER et al., 2011).
\end{abstract}


A área de estudo, anteriormente à desapropriação, era constituída por pequenas propriedades rurais de atividade agropecuária. Em virtude da presença do gado bovino, o solo tornou-se moderadamente compactado, o que foi verificado durante o preparo do plantio e pela ausência de regeneração natural na área. Na ocasião da implantação do experimento, no local predominavam espécies herbáceas, tais como Andropogon bicornis L. (Poaceae) e Eryngium horridum Malme (Apiaceae), ambas tolerantes a solos de baixa fertilidade, ácidos e antropizados (LORENZI, 2000).

\section{Seleção de espécies}

As espécies arbóreas utilizadas no experimento foram previamente selecionadas com base em levantamento fitossociológico de uma área de referência e de outras em processo de restauração, todas próximas da área de estudo (MARCUZZO, 2012; MARCUZZO et al., 2013). Além disso, utilizou-se como critério de seleção a definição de dois grupos de plantio, formados por espécies de preenchimento que apresentam rápido crescimento e densa cobertura de copa e por espécies de diversidade, que abrangem todos os grupos sucessionais (pioneiras, secundárias e tardias) (GANDOLFI et al., 2007; NAVE; RODRIGUES, 2007).

Para a definição dos grupos, foram utilizados trabalhos de avaliação do crescimento de espécies em reflorestamentos, bem como suas características de desenvolvimento em fases iniciais de projetos de restauração (SOUZA et al., 2001; CHADA et al., 2004; MELO; DURIGAN, 2007; ANDREAZZA et al., 2008; NÓBREGA et al., 2008; SOUZA; PIÑA-RODRIGUES; 2013).

Utilizou-se um total de 13 espécies arbóreas no arranjo. Assim, o grupo de preenchimento, com seis espécies, foi formado por Enterolobium contortisiliquum (Vell.) Morong., Parapiptadenia rigida (Benth.) Brenan, Inga vera Willd, Schinus terebinthifolius Raddi, Luehea divaricata Mart. e Psidium cattleianum L. O grupo de diversidade, com sete espécies, foi composto por Eugenia uniflora L., Allophylus edulis (A. St.-Hil., Cambess. \& A. Juss.) Radlk., Cedrela fissilis Vell., Prunus myrtifolia (L.) Urb., Jacaranda micrantha Cham., Cupania vernalis Cambess. e Cabralea canjerana (Vell.) Mart.

A leguminosa Vicia sativa L. (ervilhaca) foi selecionada para ser introduzida nos tratamentos como cobertura do solo por ser uma planta forrageira de inverno, ocasião da implantação do experimento, além do fato de apresentar alta capacidade de fixar nitrogênio, conforme observado por Giacomini et al. (2003).

\section{Delineamento experimental}

$\mathrm{O}$ experimento foi implantado em delineamento em blocos ao acaso, seguindo arranjo fatorial $2 \times 2 \times 4$. O fator A representou os níveis de preparo de solo (cova e subsolagem), o fator B a presença ou ausência da leguminosa Vicia sativa e o fator C as quatro ocasiões de avaliação, considerando parcelas subdivididas no tempo, com quatro repetições por tratamento. Dessa forma, os tratamentos foram constituídos por: $\mathrm{T} 1$ = cova + espécies arbóreas (grupo de preenchimento - GP e grupo de diversidade GD); T2 = cova + espécies arbóreas (GP + GD) com Vicia sativa; T3 = subsolagem + espécies arbóreas $(\mathrm{GP}+\mathrm{GD}) ;$ e T4 = subsolagem + plantio de espécies arbóreas $(\mathrm{GP}+\mathrm{GD})$ com Vicia sativa .

Cada parcela apresentou 19,5 x 19,5 m, com mudas dispostas em linhas com espaçamento de 1,5 x 1,5 m (169 indivíduos), mantendo-se a distância de 0,5 m entre parcelas. Cada espécie foi representada por 13 indivíduos na parcela, tanto do grupo de preenchimento (seis espécies) como de diversidade (sete espécies). No total, foram plantadas 2.704 mudas em $6.400 \mathrm{~m}^{2}$ (16 parcelas).

\section{Implantação do experimento}

Inicialmente foi realizada uma roçada na área total, para a marcação das parcelas e sorteio dos tratamentos. No tratamento 1 foram abertas covas $\left(\cong 30 \mathrm{~cm}^{3}\right)$ com pá de corte, para o plantio das mudas, conforme a posição e o número de espécies definidos pelos grupos de preenchimento e diversidade. $\mathrm{O}$ mesmo procedimento foi realizado no tratamento 2 .

Para os tratamentos 3 e 4 , o preparo do solo foi por subsolagem. As linhas de plantio foram preparadas com uma haste acoplada a um trator agrícola com sulcos de $30 \mathrm{~cm}$, distanciados $1,5 \mathrm{~m}$ entre si. Posteriormente, no ponto de plantio foi aberta pequena cova do tamanho do torrão. As mudas utilizadas foram produzidas em tubete de $110 \mathrm{~cm}^{3}$, contendo substrato à base de turfa, sendo que no momento do plantio elas apresentavam em torno de $22 \mathrm{~cm}$ de altura. Em todos os tratamentos, cada muda recebeu cerca de $5 \mathrm{~kg}$ de composto orgânico, o qual foi distribuído ao longo da linha.

Nos tratamentos 2 e 4, a ervilhaca foi semeada a lanço no momento do plantio, totalizando $2,6 \mathrm{~kg}$ de sementes por parcela. 


\section{Coleta e análise dos dados}

Durante o experimento, implantado em março de 2010 (período de outono sem ocorrência de temperaturas extremas), foram realizadas quatro avaliações (setembro/2010, março/2011, setembro/2011, março/2012), obtendo-se as seguintes variáveis: percentual de mortalidade; altura da parte aérea das mudas $(\mathrm{cm})$, desde o coleto até a gema apical, mensurada com régua graduada; e diâmetro do coleto $(\mathrm{mm})$, medido com paquímetro.

Aos 24 meses de observação, foi realizada a medição do grau de sombreamento, por meio da projeção da copa, a qual foi medida através da distância entre o tronco e a maior projeção da copa, nos quatros sentidos cardeais. Esse procedimento permitiu a obtenção da fração do solo sombreada, representada pela somatória da parte aérea de cada árvore nos diferentes tratamentos, expressa em porcentagem, calculada pela expressão $\mathrm{C}=100{ }^{\mathrm{n}} \sum_{\mathrm{i}=1} \mathrm{Ci} / \mathrm{A}$, em que $\mathrm{C}=$ grau de cobertura $(\%), \mathrm{Ci}=$ área da projeção da copa do indivíduo i $\left(\pi\right.$. $\left.\mathrm{Di}^{2} / 4\right)$, Di $=$ diâmetro médio da copa do indivíduo i $(\mathrm{m})$, $\mathrm{n}=$ número de indivíduos medidos na área $\mathrm{A}$ e $\mathrm{A}=$ área da parcela útil $\left(\mathrm{m}^{2}\right)$ (DURIGAN; SILVEIRA, 1999).

Para verificar diferenças entre os tratamentos, os dados de altura, diâmetro do coleto e mortalidade das mudas foram submetidos à análise de variância. Quando necessário, realizou-se o desdobramento das interações, seguido pela análise de regressão polinomial, a 5\% de probabilidade de erro. No caso de diferença significativa de equações quadráticas, determinou-se o ponto crítico (PC). Nas análises, utilizou-se o software estatístico SISVAR (FERREIRA, 2008). Para análise do potencial de desenvolvimento das espécies plantadas, utilizou-se o teste de Kruskal-Wallis, que foi realizado por meio da ordenação de $k$ amostras, obtendo-se o posto médio $(\mathrm{Pm})$ de cada espécie. Posteriormente, os Pm das variáveis altura $(\mathrm{H})$, diâmetro do coleto $(\mathrm{D})$, diâmetro de projeção da copa $(\mathrm{Dp})$ e percentual de mortalidade (Mor) foram comparados pelo teste de Dunn (CALLEGARI-JACQUES, 2003). Cada grupo (preenchimento e diversidade) foi analisado separadamente, tendo em vista sua diferente funcionalidade.

\section{RESULTADOS E DISCUSSÃO}

A subsolagem na linha de plantio proporcionou melhor crescimento no diâmetro do coleto $(8,45 \mathrm{~mm})$ das espécies plantadas ( $\mathrm{p}<0,05)$, em relação às mudas plantadas sem subsolagem $(7,43 \mathrm{~mm})$. As demais variáveis não apresentaram significância quanto ao preparo do solo e o uso de leguminosa.

A subsolagem pode ser descrita como a ação de romper camadas adensadas e compactadas de solo (SASAKI; GONÇALVES, 2005). Considerando que na área de estudo o solo encontrava-se alterado, a subsolagem reduziu a densidade do solo, melhorando as condições de aeração e drenagem, o que favoreceu o desenvolvimento do diâmetro do coleto. Essa característica é favorável, pois, conforme Ritchie et al. (2010), o aumento do diâmetro do coleto apresenta relação positiva com o sistema radicular das mudas, favorecendo seu estabelecimento.

Outros estudos apresentam resultados que confirmam a influência da subsolagem no desenvolvimento das plantas, como o de Stape et al. (2002), que observaram valores maiores para altura em mudas de eucaliptos em dois sítios preparados com subsolagem, quando comparados a áreas preparadas somente com coveamento. Chabaribery et al. (2008) destacaram a subsolagem como um dos métodos para plantio em recomposição de mata ciliar na microbacia do Córrego do Barreiro, São Paulo, assim como Faria et al. (1997), que testaram o desempenho de nove espécies florestais nativas, utilizando subsolagem no preparo do solo. Porém poucos estudos abordam a importância do uso da subsolagem no desenvolvimento de espécies florestais nativas, entre os quais Archuleta e Baxter (2008), que verificaram a eficiência da subsolagem em plantios de espécies de leguminosas nativas arbustivas em projetos de recuperação de áreas degradadas, havendo um aumento da atividade biológica, melhora da estrutura do solo e redução da erosão superficial.

Adicionalmente, Baptista e Levien (2010) descreveram que, em geral, há relação positiva entre o volume de solo preparado e o crescimento das árvores, observando biomassa aérea de Eucalyptus saligna $\mathrm{Sm}$. cinco vezes maior no tratamento com subsolagem do que por meio de coveamento mecânico, após 11 meses do plantio. Tais resultados confirmam que o preparo do solo por subsolagem pode beneficiar a planta, como foi o caso observado para o maior diâmetro médio obtido para as espécies estudadas. 
No decorrer do tempo, considerando a média geral de todos os tratamentos avaliados, houve diferença significativa para altura $(\mathrm{p}<0,0001)$, diâmetro do coleto $(\mathrm{p}<0,0001)$ e mortalidade $(\mathrm{p}<0,0001)$ das espécies plantadas.

O percentual de mortalidade apresentou comportamento quadrático crescente, com ponto crítico aos 21 meses, ocasião em que a taxa de mortalidade das plantas atingiu o seu pico (Figura 1).

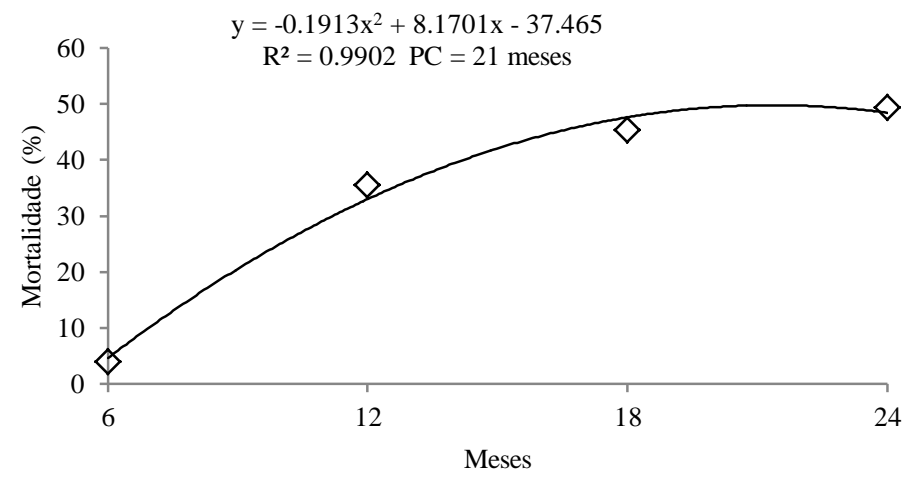

Figura 1. Percentual geral de mortalidade de mudas, durante 24 meses, em área experimental de restauração no Parque Estadual Quarta Colônia, RS.

Figure 1. Mortality rate during 24 months in experimental area of restoration in State Park Quarta Colônia, RS.

A altura também apresentou comportamento quadrático crescente no decorrer do tempo (Figura 2A), com ponto crítico aos 25 meses. Esse resultado pode ter ocorrido devido à competição das mudas com a ervilhaca presente na área. Destaca-se que a ervilhaca somente foi usada como cobertura, e talvez por isso tenha ocupado o papel de competidora, uma vez que a mesma não foi incorporada ao solo.
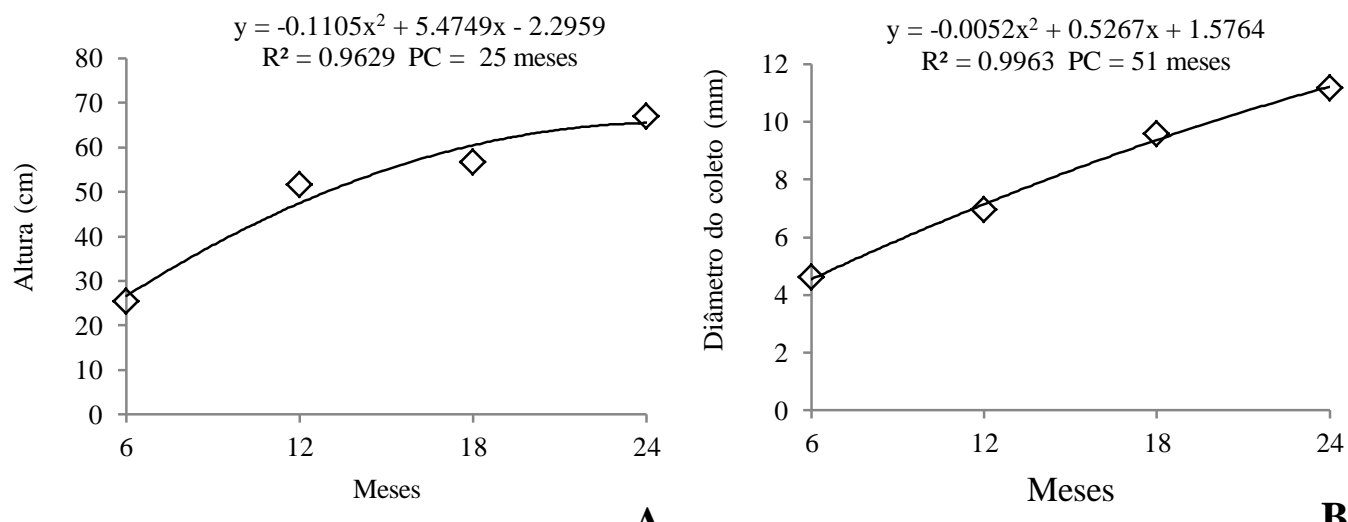

Figura 2. Médias gerais de crescimento em altura $(\mathrm{cm})(\mathrm{A})$ e diâmetro do coleto $(\mathrm{mm})(\mathrm{B})$ das espécies arbóreas ao longo de 24 meses, em área experimental de restauração no Parque Estadual Quarta Colônia, RS.

Figure 2. Growth in height (cm) (A) and collar diameter (mm) (B) of tree species over 24 months, in experimental area of restoration in Quarta Colônia State Park, RS.

Para o diâmetro, o crescimento também foi quadrático crescente (Figura 2B), proporcionado pela disponibilidade de espaço para o crescimento das plantas. No entanto, o ponto crítico para essa variável extrapolou consideravelmente (51 meses) o tempo de observação, comprometendo resultados mais conclusivos. 
Todas as espécies de preenchimento apresentaram um percentual de mortalidade maior que $10 \%$ (Tabela 1), o que pode ter ocorrido devido ao tamanho das mudas conduzidas no campo, pois, conforme RITCHIE et al. (2010), sob condição ambiental menos favorável, com mato-competição, devem-se priorizar mudas de maior dimensão $(>30 \mathrm{~cm})$. A maior taxa de mortalidade foi de Parapiptadenia rigida (angico-vermelho), com 31,7\%, e a menor foi de Luehea divaricata (açoita-cavalo), com 11,5\%. Entretanto, as espécies foram consideradas estatisticamente semelhantes quanto à mortalidade (Tabela 1).

Tabela 1. Percentual de mortalidade (Mor), altura da parte aérea (H), diâmetro do coleto (D) e diâmetro de copa (DP) de espécies nativas de preenchimento após 24 meses de implantação, utilizadas na recuperação de área degradada, Parque Estadual Quarta Colônia, RS.

Table 1. Percentage of mortality (Mor), above ground height $(\mathrm{H})$, collect diameter (D) and Cup diameter $(\mathrm{Dp})$ of native species used in recovering degraded area, 24 months after planting, Quarta Colônia State Park, RS.

\begin{tabular}{lcccccccc}
\hline $\begin{array}{l}\text { Grupo de } \\
\text { preenchimento }\end{array}$ & $\begin{array}{c}\text { Mor } \pm \text { sd } \\
(\%)\end{array}$ & $\begin{array}{c}\text { Pm } \\
\text { Mor }\end{array}$ & $\begin{array}{c}\text { H } \\
(\mathbf{c m})\end{array}$ & $\begin{array}{c}\text { Pm } \\
\mathbf{H}\end{array}$ & $\begin{array}{c}\mathbf{D} \\
(\mathbf{m m})\end{array}$ & $\begin{array}{c}\text { Pm } \\
\mathbf{D}\end{array}$ & $\begin{array}{c}\text { Dp } \\
(\mathbf{c m})\end{array}$ & $\begin{array}{c}\text { Pm } \\
\mathbf{D p}\end{array}$ \\
\hline Enterolobium contortisiliquum & $23,1 \pm 11,7$ & $\mathrm{a}$ & 193,4 & $\mathrm{a}$ & 41,3 & $\mathrm{a}$ & 41,0 & $\mathrm{a}$ \\
Schinus terebinthifolius & $25,0 \pm 25,9$ & $\mathrm{a}$ & 109,0 & $\mathrm{ab}$ & 15,0 & $\mathrm{bc}$ & 29,0 & $\mathrm{~b}$ \\
Parapiptadenia rigida & $31,7 \pm 16,2$ & $\mathrm{a}$ & 97,8 & $\mathrm{ab}$ & 11,9 & $\mathrm{bc}$ & 18,8 & $\mathrm{bc}$ \\
Luehea divaricata & $11,5 \pm 7,1$ & $\mathrm{a}$ & 94,7 & $\mathrm{bc}$ & 16,6 & $\mathrm{~b}$ & 21,5 & $\mathrm{bc}$ \\
Inga vera & $19,2 \pm 14,2$ & $\mathrm{a}$ & 91,8 & $\mathrm{bc}$ & 17,1 & $\mathrm{~b}$ & 18,2 & $\mathrm{bc}$ \\
Psidium cattleianum & $16,3 \pm 11,9$ & $\mathrm{a}$ & 55,0 & $\mathrm{c}$ & 9,1 & $\mathrm{c}$ & 15,8 & $\mathrm{c}$ \\
\hline
\end{tabular}

Sd: desvio padrão. Médias seguidas de letras diferentes são significativamente diferentes pelo teste de Kruskal-Wallis $(\mathrm{p}<0.05)$. Comparação do posto médio pelo Teste de Dunn (Pm) e valores de parâmetros morfológicos médios.

A elevada mortalidade das espécies pode ser decorrente da época de plantio (outono), em ano de inverno com fortes geadas seguido de verão rigoroso, e da presença de Vicia sativa nos tratamentos 3 e 4, nos quais se pôde observar que a ervilhaca cobriu completamente as mudas das espécies arbóreas, competindo por recursos como água, nutrientes e, principalmente, luz. Esse fato seria superado se a ervilhaca fosse plantada com antecedência e antes do plantio das arbóreas fosse incorporada ao solo.

Beltrame e Rodrigues (2008) avaliaram outra leguminosa, Cajanus cajan (L.) Millsp., associada a espécies florestais, sendo que, no período de 22 e de 34 meses após o plantio, ela ocasionou o aumento da mortalidade, tanto de espécies pioneiras quanto de não pioneiras, o que ocorreu principalmente nos tratamentos em que a densidade da leguminosa foi maior. As principais vantagens das plantas de cobertura em relação ao cultivo isolado consistem no maior rendimento de matéria seca, no acúmulo de nutrientes e na fixação biológica de $\mathrm{N}_{2}$ pelas leguminosas (HEINRICHS et al., 2001). Dessa forma, a ervilhaca poderia levar a resultados positivos se manejada no experimento, através de corte e incorporação ao solo antes do plantio, prática essa que não foi utilizada no presente estudo.

Enterolobium contortisiliquum (timbaúva) mostrou-se com elevado potencial como espécie de preenchimento (Tabela 1), tendo em vista que, juntamente com Schinus terebinthifolius (pimenteira) e Parapiptadenia rigida (angico-vermelho), cresce rapidamente em altura (Figura 3A), tendo capacidade de superar a mato-competição. Essa espécie, juntamente com Inga vera (ingá-banana) e Schinus terebinthifolius, apresenta maior diâmetro do coleto, sugerindo a formação de um sistema radicular mais amplo, capaz de explorar os recursos do solo (GONÇALVES, 2002). Outros autores obtiveram resultados semelhantes em relação ao diâmetro e à altura de timbaúva (FERREIRA et al., 2007) e pimenteira (SOUZA et al., 2001), em estudos abordando o desenvolvimento de espécies nativas em áreas degradadas.

Com base nos valores médios do diâmetro de copa e do percentual de mortalidade das espécies (Tabela 1), observou-se que, após dois anos de implantação, nas circunstâncias do experimento, somente cerca de $20 \%$ do solo estava coberto pelo grupo de preenchimento, reduzindo as chances de desenvolvimento das "espécies de diversidade". As espécies de preenchimento utilizadas, apesar de apresentarem crescimento rápido, têm menor incremento na região Sul do Brasil quando comparada às outras regiões, além de, possivelmente, terem sofrido influência negativa de fatores exógenos, descritos anteriormente.

Conforme Melo e Durigan (2007), a qualidade de sítio, principalmente no que se refere às características de solo, pode ser responsável pelas diferenças nos índices de cobertura, para 
reflorestamentos com idades semelhantes. Entretanto, outros fatores também responsáveis pelo desenvolvimento das espécies, como baixas temperaturas e curto fotoperíodo, os quais desfavorecem o desenvolvimento das espécies durante o inverno na região Sul do Brasil, restringem a utilização de modelos aplicados em outras regiões climáticas do país. Assim, modelos previamente concebidos para o processo de restauração dependem de adequações às condições ambientais regionais.

Entre as espécies de preenchimento estudadas, Psidium cattleianum (araçá), apesar de ter apresentado crescimento inferior em relação às demais (Figura 3), é considerada uma espécie com funcionalidade importante, devido à sua síndrome de polinização (entomofilia) e dispersão (ornitocoria), ou seja, é uma espécie que atrai a fauna e contribui para o ingresso de outras espécies no sistema. No entanto, sua utilização no grupo de preenchimento deve ser avaliada, pois, caso se busque uma rápida cobertura vegetal, não seria interessante incluí-la nesse grupo, mas sim no grupo de diversidade.

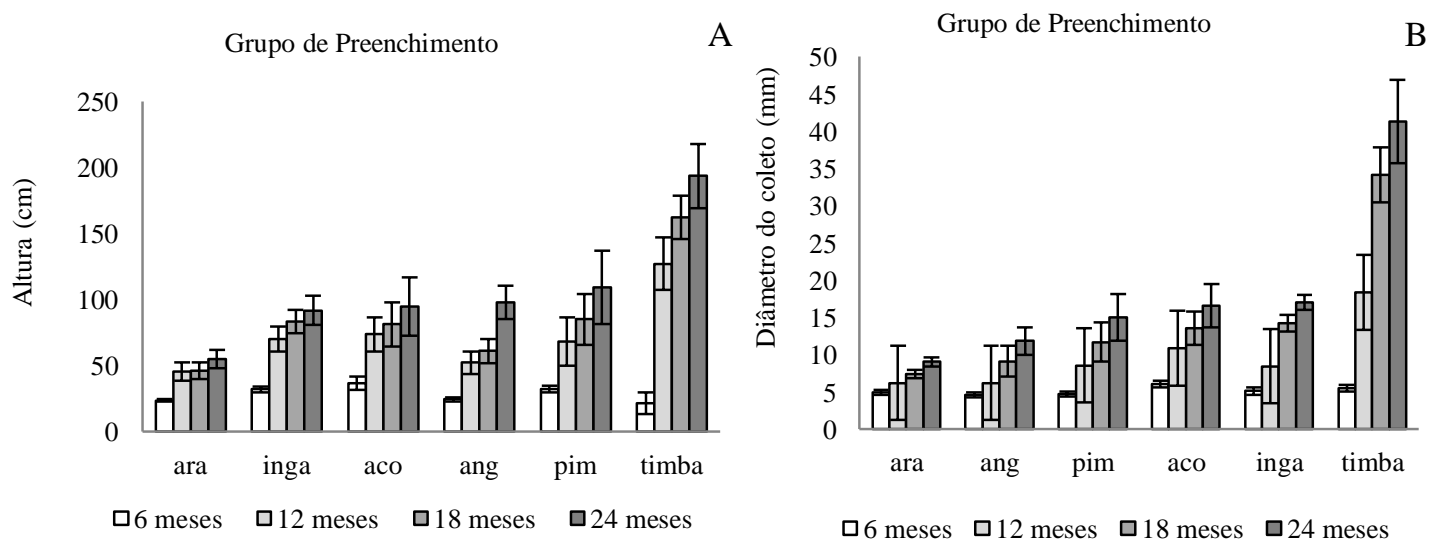

Figura 3. Crescimento médio no tempo em altura $(\mathrm{cm})(\mathrm{A})$ e diâmetro $(\mathrm{mm})(\mathrm{B})$ da média dos indivíduos do grupo de preenchimento, independentemente do método de preparo do solo.

Figure 3. Average growth in time in height $(\mathrm{cm})(\mathrm{A})$ and diameter $(\mathrm{mm})(\mathrm{B})$ of the average fill group, regardless of the method of soil preparation.

Nota: ara: Psidium cattleianum; inga: Inga vera; aco: Luehea divaricata; ang: Parapiptadenia rigida; pim: Schinus terebinthifolius; timba: Enterolobium contortisiliquum.

O grupo de diversidade, quando comparado ao grupo de preenchimento, apresentou menor desempenho em relação às variáveis de altura, diâmetro do coleto e cobertura de copa, como esperado. Esses resultados correspondem às diferenças ecofisiológicas das espécies consideradas pioneiras, secundárias iniciais e tardias, além da influência da cobertura do dossel no estabelecimento das espécies mais tardias do processo sucessional, uma vez que a cobertura de copa na área, possivelmente, não foi suficiente para criar condições favoráveis de intensidade luminosa, temperatura e umidade.

As espécies que apresentaram maior tolerância às condições da área no período considerado são Eugenia uniflora (pitangueira) e Allophylus edulis (chal-chal), com menor percentual de mortalidade, associado a razoável crescimento em altura (Tabela 2). Isso, provavelmente, ocorreu devido às condições mais favoráveis de umidade do solo e tolerância maior a condições de maior luminosidade, pois, conforme Backes e Irgang (2002), essas espécies são indicadas para plantios no entorno de nascentes, rios e reservatórios. Muitas espécies, como Trema micrantha, citada por Moraes et al. (2006), apresentam baixa mortalidade e elevado crescimento na fase inicial de desenvolvimento, bem como ciclo de vida de 10-20 anos.

Adicionalmente, Cedrela fissilis (cedro), embora com alto percentual de mortalidade, apresentou os maiores valores em diâmetro de coleto (Tabela 2) e copa, embora sem diferença significativa em relação às outras espécies desse grupo. Resultado semelhante foi encontrado por Melotto et al. (2009), que observou o cedro como sendo a segunda espécie com maior crescimento em diâmetro do coleto em sistemas silvipastoris. Em plantios de restauração de 2,5 anos, em Ribeirão Grande, SP, Nave e Rodrigues (2007) encontraram indivíduos de cedro com $50 \mathrm{~cm}$ de cobertura de copa. 
Tabela 2. Percentual de mortalidade (Mor), altura da parte aérea (H), diâmetro do coleto (D) e diâmetro de copa (DP) de espécies de diversidade utilizadas na recuperação de área degradada, Parque Estadual Quarta Colônia, RS.

Table 2. Percentage of mortality (Mor), above ground height $(\mathrm{H})$, collect diameter (D) and crown diameter (Dp) of species used in the recovery of degraded area, Quarta Colônia State Park, RS.

\begin{tabular}{lcccccccc}
\hline $\begin{array}{l}\text { Grupo de } \\
\text { diversidade }\end{array}$ & $\begin{array}{c}\text { Mor } \mathbf{m} \mathbf{s d} \\
(\mathbf{\%})\end{array}$ & $\begin{array}{c}\text { Pm } \\
\text { Mor }\end{array}$ & $\begin{array}{c}\text { H } \\
(\mathbf{c m})\end{array}$ & $\begin{array}{c}\text { Pm } \\
\mathbf{H}\end{array}$ & $\begin{array}{c}\mathbf{D} \\
(\mathbf{m m})\end{array}$ & $\begin{array}{c}\text { Pm } \\
\mathbf{D}\end{array}$ & $\begin{array}{c}\text { Dp } \\
(\mathbf{c m})\end{array}$ & $\begin{array}{c}\text { Pm } \\
\mathbf{D p}\end{array}$ \\
\hline Eugenia uniflora & $32,2 \pm 23,0$ & $\mathrm{a}$ & 48,7 & $\mathrm{a}$ & 4,4 & $\mathrm{ab}$ & 15 & $\mathrm{ab}$ \\
Allophylus edulis & $35,0 \pm 16,4$ & $\mathrm{a}$ & 47,7 & $\mathrm{a}$ & 6,2 & $\mathrm{bc}$ & 12 & $\mathrm{~b}$ \\
Cedrela fissilis & $68,2 \pm 17,3$ & $\mathrm{~b}$ & 53,4 & $\mathrm{a}$ & 17,1 & $\mathrm{bc}$ & 26,5 & $\mathrm{a}$ \\
Prunus myrtifolia & $86,7 \pm 3,98$ & $\mathrm{~b}$ & 68,5 & $\mathrm{a}$ & 8,8 & $\mathrm{~b}$ & 10 & $\mathrm{~b}$ \\
Jacaranda micrantha & $80,0 \pm 11,5$ & $\mathrm{bc}$ & 34,7 & $\mathrm{a}$ & 8,8 & $\mathrm{ab}$ & 0 & $\mathrm{c}$ \\
Cupania vernalis & $76,7 \pm 37,6$ & $\mathrm{bc}$ & 28,6 & $\mathrm{a}$ & 4,3 & $\mathrm{c}$ & 0 & $\mathrm{c}$ \\
Cabralea canjerana & $98,5 \pm 5,4$ & $\mathrm{c}$ & 30,0 & $\mathrm{~b}$ & 5,2 & $\mathrm{a}$ & 0 & $\mathrm{c}$ \\
\hline
\end{tabular}

Sd: desvio padrão; método de Dunn; médias seguidas de letras diferentes são significativamente diferentes pelo teste de Kruskalwallis $(\mathrm{P}<0.05)$; comparação do posto médio pelo Teste de Dunn $(\mathrm{Pm})$.

Prunus myrtifolia (pessegueiro-bravo) apresentou maior valor em altura (Figura 4), porém uma das maiores taxas de mortalidade, o que demonstra que essa espécie deve ser introduzida em uma fase posterior, quando houver uma maior cobertura de dossel e tiver sido incorporada a leguminosa ao solo. A mesma recomendação é indicada para Cupania vernalis (camboatá-vermelho), Jacaranda micrantha (caroba) e Cabralea canjerana (canjerana).

Entretanto, conforme Brancalion et al. (2010), a utilização inicial de espécies pioneiras e tardias de dossel e subdossel, dispostas em arranjo espacial favorável e com abundância adequada, pode favorecer a formação de um dossel predominante de pioneiras em apenas dois anos. Os resultados verificados no experimento apontam a necessidade de maior tempo para o fechamento de dossel.

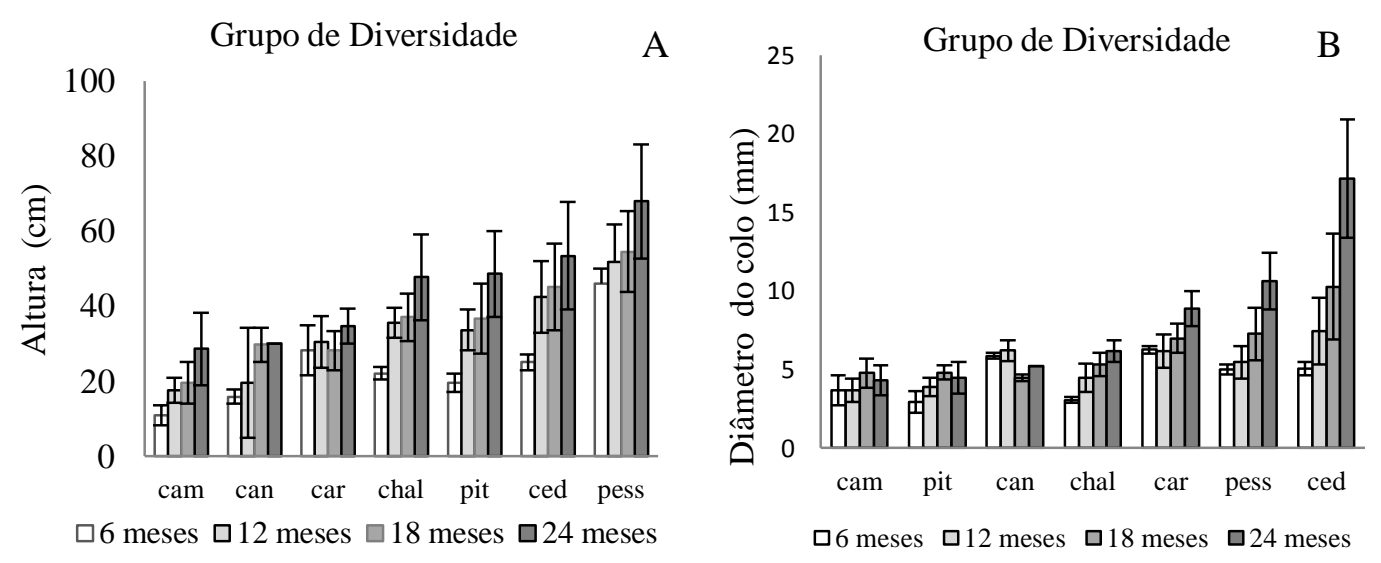

Figura 4. Crescimento médio no tempo em altura $(\mathrm{cm})(\mathrm{A})$ e diâmetro $(\mathrm{mm})(\mathrm{B})$ da média dos indivíduos do grupo de diversidade, independentemente do método de preparo do solo.

Figure 4. Average growth along the time in height $(\mathrm{cm})(A)$ and diameter $(\mathrm{mm})(B)$ of the average of the individuals in the diversity group, regardless of the method of soil preparation.

Nota: cam: Cupania vernalis; can: Cabralea canjerana; car: Jacaranda micrantha; chal: Allophylus edulis; pit: Eugenia uniflora; ced: Cedrela fissilis; pess: Prunus myrtifolia.

Assim, nas circunstâncias deste estudo, indica-se que a implantação do grupo de diversidade deverá ocorrer sob maior cobertura do dossel. Para áreas degradadas em Florestas Estacionais Subtropicais no sul do Brasil, isso possivelmente ocorre devido às condições de baixas temperaturas em determinado período do ano, cujo desenvolvimento da vegetação é mais lento, gerando maior demanda de tempo para o desenvolvimento do grupo de preenchimento. Tendo em vista tais circunstâncias, sugere-se 
que o grupo de diversidade seja plantado, a partir de dois anos, no período de primavera, em que a área irá apresentar um melhor fechamento de dossel por parte dos indivíduos plantados, oferecendo uma condição ecológica e ambiental mais adequada às espécies do grupo de diversidade.

\section{CONCLUSÕES}

- A subsolagem é um método de preparo do solo eficiente e prático para o rápido crescimento em diâmetro de espécies florestais nativas.

- O uso da leguminosa Vicia sativa não é eficiente como cobertura ou para favorecer o desenvolvimento das mudas, uma vez que não é incorporada no solo.

- Enterolobium contortisiliquum, Schinus terebinthifolius e Inga vera apresentam elevado crescimento em altura e diâmetro, características adequadas para espécies a serem utilizadas no grupo de preenchimento.

- As espécies do grupo de diversidade, nas circunstâncias do experimento, não foram adequadas para enriquecer a área.

\section{REFERÊNCIAS}

ANDREAZZA, R.; ANTONIOLLI, Z. I.; OLIVEIRA, V. L.; LEAL, L. T.; MORO JR., C.; PIENIZ, S. Ocorrência de associação micorrízica em seis essências florestais nativas do estado do Rio Grande do Sul. Revista Ciência Florestal, Santa Maria, v. 18. n. 3, p. 339 - 346, 2008.

ARCHUTELA, J. G.; BAXTER, E. S. Subsoiling promotes native plant establishment on compacted forest sites. Native Plants Journal, v. 9, n. 2, p. 117 - 122, 2008.

BACKES, P.; IRGANG, B. Árvores do sul: guia de identificação e interesse ecológico. Santa Cruz do Sul: Instituto Souza Cruz, 2002. 325 p.

BAPTISTA, J.; LEVIEN, R. Métodos de preparo de solo e sua influência na erosão hídrica e no acúmulo de biomassa da parte aérea de Eucalyptus saligna em um cambissolo háplico da depressão central do Rio Grande do Sul. Revista Árvore, Viçosa, v. 34, p. 567 - 575, 2010.

BARBOSA, L. M. Considerações gerais e modelos de recuperação de formações ciliares. In: RODRIGUES, R. R.; LEITÃO FILHO, H. F. (Ed.). Matas Ciliares: conservação e recuperação. São Paulo, 2000, p. 235 - 247.

BELTRAME, P. T.; RODRIGUES, E. Comparação de diferentes densidades de feijão-guandu (Cajanus cajan (L.) Millsp.) na restauração florestal de uma área de reserva legal no Pontal do Paranapanema, SP. Scientia Forestalis, Piracicaba, v. 36, n. 80, p. 317 - 327, 2008.

BRANCALION, H. P.; RODRIGUES, R. R.; GANDOLFI, S.; KAGEYAMA, Y. P.; NAVE, G. A.; GANDARA, B. F.; BARBOSA, L. M.; TABARELLI, M. Instrumentos legais podem contribuir para a restauração de florestas tropicais diversas. Revista Árvore, Viçosa, v. 34, n. 3, p. 455 - 470, 2010.

BRITEZ, M. R.; ALGER, K.; BAUMGARTEN, E. J.; CASTELLA, R. P.; CULLEN JR., L.; FARIA, M. D.; FELFILI, J.; FERNANDES, U. R.; FONSECA, B. A. G.; LANDAU, C. E.; LIMA, F. J.; MORATO, I. M.; ORTIZ, V. J.; PADUA, V. C.; PADUA, M. S.; RADOMSKI, I. M.; SAMPAIO, B. A. Manejo do entorno. In: Fragmentação de ecossistemas, causas, efeitos sobre a biodiversidade e recomendações de políticas públicas. Brasília: Ministério do Meio Ambiente, 2003, p. 348 - 365.

CALLEGARI-JACQUES, S. M. Bioestatística: princípios e aplicações. Porto Alegre: Ed. Artmed, 2003. $255 \mathrm{p}$.

CHABARIBERY, D.; SILVA, J. R. da; TAVAREZ, L. F. de J.; LOLI, M. V. B.; SILVA, M. R. da; MONTEIRO, A. V. V. M. Recuperação de matas ciliares: sistemas de formação de floresta nativa em propriedades familiares. Informações Econômicas, São Paulo, v. 38, n. 6, p. 07 - 20, 2008. 
CHADA, S. S.; CAMPELO, E. F. C.; FARIA, S. M. Sucessão vegetal em uma encosta reflorestada com leguminosas arbóreas em Angra dos Reis, RJ. Revista Árvore, Viçosa, v. 28, n. 6, p. 801 - 809, 2004.

DURIGAN, G.; SILVEIRA, E. R. Recomposição de mata ciliar em domínio de cerrado, Assis, SP. Scientia Forestalis, Piracicaba, n. 56, p. 15 - 28, 1999.

EMPRESA BRASILEIRA DE PESQUISA AGROPECUÁRIA (EMBRAPA). Centro Nacional de Pesquisa de Solos. Sistema brasileiro de classificação de solos. 2. ed. Rio de Janeiro: EMBRAPA, 2006.

FARIA, J. M. R.; DAVID, A. D.; BOTELHO, S. A. Comportamento de duas espécies florestais em área degradada, com adubações de plantio. Revista Cerne, Lavras, MG, v. 3, n. 1, p. 1997.

FERREIRA, D. F. SISVAR: um programa para análises e ensino de estatística. Revista Symposium. Lavras, v. 6, p. 36 - 41, 2008.

FERREIRA, R. A.; DAVIDE, A. C.; BEARZOTI, E.; MOTTA, M. S. Semeadura direta com espécies arbóreas para recuperação de ecossistemas florestais. Cerne, Lavras, v. 13, n. 3, p. 271 - 279, 2007.

GANDOLFI, S.; RODRIGUES, R. R.; MARTINS, S. V. Theoretical bases of the forest ecological restoration. In: RODRIGUES, R. R.; MARTINS S. V.; GANDOLFI, S. (Ed.). High diversity forest restoration in degraded áreas. New York: Nova Science Publishers, 2007. 247 p.

GIACOMINI, S. J.; AITA, C.; HÜBNER, P. A.; LUNKES, A.; GUIDINI, A.; AMARAL, B. E. Liberação de fósforo e potássio durante a decomposição de resíduos culturais em plantio direto. Pesquisa Agropecuária Brasileira, v. 38, n. 9, Brasília, p. 1097 - 1104, 2003.

GONÇALVES, J. L. M. Conservação do solo. In: GONÇALVES, J. L. M.; STAPE, J. L. (Ed.) Conservação e cultivo de solos para plantações florestais. Piracicaba: IPEF, 2002. p. 47 - 129.

HEINRICHS, R.; AITA, C.; AMADO, T. J. C.; FANCELLI, A. L. Cultivo consorciado de aveia e ervilhaca: relação $\mathrm{C} / \mathrm{N}$ da fitomassa e produtividade do milho em sucessão. Revista Brasileira de Ciência do Solo, v. 25, p. 331 - 340, 2001.

INSTITUTO BRASILEIRO DE GEOGRAFIA E ESTATÍSTICA (IBGE). 2012. Manual técnico da vegetação brasileira. 2. ed. Disponível em: <ftp://geoftp.ibge.gov.br/documentos/recursos_naturais /manuais_tecnicos/manual_tecnico_vegetação_brasileira.pdf >.

LEITE, P. F. Contribuição ao conhecimento fitoecológico do sul do Brasil. Ciência e Ambiente, v. 24, p. $51-63,2002$.

LORENZI, H. Plantas daninhas no Brasil. Editora Plantarum, 2000. 386 p.

MELO, A. C. G.; DURIGAN, G. Evolução estrutural de reflorestamentos de restauração de mata ciliar no médio Vale do Paranapanema. Scientia Forestalis, Piracicaba, n. 73, p. 101 - 111, 2007.

MELOTTO, A.; NICODEMO, L. M.; BOCCHESE, R. A.; LAURA, A. V.; NETO, G. M. M.; SCHLEDER, D. D.; POTT, A.; SILVA, P. V. Sobrevivência e crescimento inicial em campo de espécies florestais nativas do Brasil central indicadas para sistemas silvipastoris. Revista Árvore, Viçosa, v. 33, n. 3, p. $425-432,2009$.

MILES, L.; NEWTON, A. C.; DEFIUS, R. S.; RAVILIOUS, C.; MAY, I.; BLYTH, S.; KAPOS, V. A global overview of the conservation status of tropical dry forests. Journal of Biogeography, v. 33, p. 491 - 505, 2006.

MORAES, L. F. D.; ASSUMPÇÃO, J. M.; LUCHIARI, C.; PEREIRA, T. S. Plantio de espécies arbóreas nativas para a restauração ecológica na Reserva Biológica de Poço das Antas, Rio de Janeiro, Brasil. Rodriguésia, v. 57, n. 3, p. 477 - 489, 2006.

MURPHY, P. G.; LUGO, A. E. Ecology of tropical dry forest. Annual Review of Ecology and Systematics, v. 17, p. 67 - 88, 1986. 
NAVE, A. G.; RODRIGUES, R. R. Combination of species into filling and diversity groups as forest restoration methodology. In: RODRIGUES, R. R.; MARTINS S. V. High diversity forest restoration in degraded areas. New York: Nova Science Publishers, 2007. p. 103 - 126.

NIMER, E. Clima. In: INSTITUTO BRASILEIRO DE GEOGRAFIA E ESTATÍSTICA (IBGE). Geografia do Brasil: Região Sul. Rio de Janeiro: IBGE, 1990. p. 151 - 187.

RITCHIE, G, A.; LANDIS, T. D.; DUMROESE, R. K.; HAASE, D. L. Assessing plant quality. In: The container tree nursery manual, v. 7, Seedling Processing, Storage, and Outplanting. Whashington, DC: Agric. Handbk. p. 19 - 81. 2010.

RODRIGUES, R. R.; BRANCALION, P. H. S.; ISERNHAGEN, I. Pacto pela restauração da mata atlântica: referencial dos conceitos e ações de restauração florestal. São Paulo: LERF/ESALQ: Instituto BioAtlântica, 2010. 259 p.

SASAKI, C. M.; GONÇALVES, J. L. M. Desempenho operacional de um subsolador em função da estrutura, do teor de argila e de água em três latossolos. Scientia Forestalis, Piracicaba, n. 69, 2005. p. 115 - 124.

SCHUMACHER, M. V.; LONGHI, S. J.; BRUN, E. J.; LILKA, R. V. A Floresta Estacional Subtropical: caracterização e ecologia no rebordo do Planalto Meridional. Santa Maria: [s.n.], 2011. 320 p.

SOUZA, A. P.; VENTURIN, N.; MACEDO, G. L. R.; ALVARENGA, N. I. M.; SILVA, F. V. Estabelecimento de espécies arbóreas em recuperação de área degradada pela extração de areia. Revista Cerne, v. 7, n. 2, p. 43 - 52, 2001.

SOUZA, S. C. M.; PIÑA-RODRIGUES, M. C. F. Desenvolvimento de espécies arbóreas em sistemas agroflorestais para recuperação de áreas degradadas na Floresta Ombrófila Densa, Paraty, RJ. Revista Árvore, Viçosa, v. 37, n. 1, p. 89 - 98, 2013.

STAPE, J. L.; ANDRADE, S.; GOMES, A. N.; KRIJCI, L. C.; RIBEIRO, J. A. Definições de métodos de preparo do solo para silvicultura em solos coesos do litoral norte da Bahia. In: GONÇALVES, J. L. M.; STAPE, J. L. (Ed.). Conservação e cultivo de solos para plantações florestais. Piracicaba: IPEF, 2002. p. 259 - 296.

TABARELLI, M; MANTOVANI, W. A regeneração de uma Floresta Tropical Montana após corte e queima (São Paulo, Brasil). Rev. Brasil. Biol., v. 59, n. 2, p. 239 - 250, 1999. 
FLORESTA, Curitiba, PR, v. 45, n. 1, p. 129 - 140, jan. / mar. 2015. Marcuzzo, S. B.; Araújo, M. M.; Gasparin, E. 